\title{
Calculations of dynamo coefficients in Parker unstable disks without shear
}

\begin{abstract}
K. Otmianowska-Mazur *
Astronomical Observatory, Jagiellonian University, 30-244 Kraków, Poland

Received 10 March 2003 / Accepted 16 May 2003

Abstract. We investigate the influence of the Coriolis force and magnetic reconnection on the evolution of the Parker instability in galactic disks. We apply a three-dimensional (3D) model of a local gas cube, permeated by an azimuthal regular magnetic field. We numerically solve MHD equations including the contribution of the Coriolis force. At this stage of the investigation we omit the effects of rotational shear. Our previous simulations demonstrate that Parker instability leads to the formation of helically twisted magnetic flux tubes forming a significant poloidal magnetic field component on the scale of the whole cube. Such an evolution represents an example of the fast dynamo process proposed by Parker (1992). In the present work we extend our earlier computations by calculating the basic coefficients of the MHD dynamo as time-dependent functions. The wellknown dynamo coefficients $\alpha$ and $\eta_{\mathrm{T}}$ - both in the relevant tensorial formulations - are derived from small scale gas motions present in the Parker instability model, so in a local formulation the total turbulent electromotive force (EMF) is described as a quantity dependent on time. The EMF-coefficients $\alpha$ and $\eta_{\mathrm{T}}$ are evaluated within the limit of high microscopic conductivity.
\end{abstract}

Key words. galaxies: ISM - galaxies: magnetic fields - ISM: magnetic fields - MHD

\section{Introduction}

Historically, the classical turbulent dynamo theory involved the problem of the dynamo coefficients obtained from fluctuating gas motions present in the interstellar medium (ISM) caused by different sources of turbulences like supernova (SN) explosions, winds from stars, different instabilities, shearing motions etc. The dynamo process relies on the concept of a helical turbulence, which is parameterized by the transport coefficients: $\alpha$, allowing for a magnetic field amplification, and turbulent diffusivity $\eta$ (Ruzmaikin et al. 1988) responsible for the magnetic field decay. In general, both dynamo coefficients should be treated as tensors. The first of them, $\hat{\alpha}$ can be split in two parts: the symmetric one responsible for the magnetic field amplification $\left(\alpha_{x x}=\alpha_{r r}, \alpha_{y y}=\alpha_{\phi}\right.$ and $\alpha_{z z}$, local $X, Y$ and $Z$ coordinates run along $R, \phi$ and $Z$ of the global galactic system, respectively) and the antisymmetric one representing turbulent velocities responsible for the magnetic field buoyancy (the socalled pumping effect, see also Ossendrijver et al. 2002). In a thin galactic disk an important component of the dynamo coefficient is $\alpha_{y x}$ (Ziegler 1996), also called $V_{\text {esc }}$ in Ferrière (1993a,b), presenting the magnetic field motion in the $Z$ direction (buoyancy, see Eq. (4)). The dynamo works if the buoyancy mechanism is less efficient than the magnetic field amplification. The numerical simulations made by Korpi (1999) and Ferrière (1993a,b, 1995, 1996, 1998) give values of about few $\mathrm{km} \mathrm{s}^{-1}$ for the symmetrical part of $\hat{\alpha}$ and with a two or three times higher value of $V_{\text {esc }}$. Ferrière (1998) found a ring

\footnotetext{
* e-mail: otmian@oa.uj.edu.pl
}

between certain radii in a modeled galactic disk, where the ratio $V_{\text {esc }} / \alpha_{\phi}$ is sufficiently low to allow for the magnetic field amplification. These simulations were done without back reaction of the magnetic field on the turbulent motions and the dynamo coefficients were estimated semi-analytically. Fully dynamical 3D simulations of the dynamo coefficients with a magnetic quenching of SN explosions in the local galactic medium done by Ziegler (1996) give quite opposite results. Kinematic calculations made by Ziegler et al. (1996) generally confirmed Ferrière's results. However, taking into account a magnetic quenching of turbulent motion causes a symmetrical part of $\alpha$ to be strongly suppressed by the magnetic field, while $V_{\text {esc }}$, in contrast, depends only slightly on the mean magnetic field tension. The main conclusion of this paper was that the turbulent buoyancy overwhelms any $\alpha$ action. Brandenburg et al. (1995) and Brandenburg \& Donner (1997) get strong dependence of $\alpha_{\phi}$ on the magnetic field, as well. They got the opposite sign of this coefficient than was earlier estimated in classical dynamo papers (e.g. Ruzmaikin et al. 1988). Both models of Brandenburg and his coauthors take into account strong shear so their results cannot be directly comparable to our simulations. Traditionally the problem of quenching is analyzed under the assumption that a system is in a steady state (without any time-dependence). The new simulations made by Blackman \& Brandenburg (2002) show that the assumption of the algebraic form of $\alpha$, which does not depend on time, gives inappropriate solutions for the mean magnetic field evolution. These authors take into account the time dependence of the magnetic helicity and of the functional form of the $\alpha$-quenching. They 
obtained that algebraic quenching prescriptions are inconsistent with that from a time-dependent analysis (Blackman \& Brandenburg 2002). The time-dependence of the dynamo coefficients becomes crucial in forthcoming models concerning the amplification of the mean magnetic field in the astrophysical objects. The physics of our Parker instability model incorporates the magnetic field quenching by definition.

The next important problem in the dynamo theory is a conservation of the total magnetic helicity in objects possessing high magnetic Reynolds numbers, thus also relevant for galaxies. The galactic dynamo gives the small and large-scale magnetic field with a certain value of the magnetic helicity, which should be conserved in a given volume over time (Kleeorin et al. 2003). This quantity also influences the character of the turbulent electromotive force extending the dynamo coefficients to time-dependent variables nonlinearly dependant on the magnetic field (see Kleeorin et al. 2000, 2002, 2003; Rogachevskii \& Kleeorin 2001; Kleeorin \& Rogachevskii 1999). According to this theory, the coefficient $\alpha$ separates into a magnetic and kinematic part as was originally proposed by Frisch et al. (1975) and Pouquet et al. (1976).

The mentioned above problems with the magnetic quenching of the turbulent dynamo action (summarized in Widrow 2002) prompted the idea of a new mechanism to help in solving this problem. The concept of magnetic buoyancy serving as a source of helical turbulences was considered by Parker (1992), Moss et al. (1999), Korpi et al. (1999), Brandenburg \& Schmitt (1998), Ossendrijver et al. (2001, 2002) (the last three papers studied the solar dynamo) resulting in hints that this mechanism could work both in galactic and stellar dynamos. This fast magnetic field amplification is also necessary to explain the modern observations of magnetic fields in irregular galaxies (Chyży et al. 2000, 2003; Otmianowska-Mazur et al. 2000). While the underlying physics of these models is different from the classical dynamo, the equations concerning the dynamo coefficients are the same (Widrow 2002 and references therein).

The concept of Parker instability working as the fast dynamo was considered in a series of papers by Hanasz \& Lesch (1993, 1997, 1998, 2000). They studied the dynamics of flux tubes under the influence of cosmic ray pressure with the approximation of thin tubes and estimated values of the dynamo coefficients, $\alpha$ and $\eta$. Their papers provide evidence that the Parker instability operating in rotating galactic disks contributes very efficiently to the production of the poloidal magnetic field from the initial azimuthal one. This implies that this instability can be considered as a reasonable candidate for driving the galactic $\alpha \omega$-dynamo.

In a previous paper (Hanasz et al. 2002 - Paper I) we analyzed the full 3D MHD evolution of the Parker instability in a local galactic cube, permeated by an azimuthal large-scale magnetic field and we solved numerically the 3D MHD equations including the contribution of the Coriolis force. We do not apply the process of shearing caused by differential rotation of the galactic disk, which will be the subject of a future study.

In this paper we present results of computations of the dynamo coefficients for simulations of the Parker instability in galactic disks published in Paper I, following the method described by Otmianowska-Mazur et al. (1997). In the present approach we calculate the dynamo coefficients $\hat{\alpha}$ and $\hat{\eta}$ as timedependent tensors (Ruzmaikin et al. 1988; Moffatt 1978). The possibility of stochastic excitation of magnetic fields by a fluctuating $\alpha$-effect is well established in the solar dynamo theory (e.g. Hoyng et al. 1994). These excitations may contribute, for instance, to irregularities of the solar cycle (Choudhuri 1992; Moss et al. 1992; Schmitt 1993). Numerical computations of the evolution of helical turbulences in the local medium show that despite the strong fluctuating nature, a significant $\alpha$-effect appears to be present in such flows (e.g. Brummell et al. 1998; Schmalz \& Stix 1991; Covas et al. 1997, 1998), which could be responsible for the classical large-scale dynamo action.

The numerical and theoretical studies concerning the MHD dynamo process, where we analyze what kind of gas motions could be responsible for the magnetic field amplification in astrophysical bodies, can be loosely divided into two groups: flows exhibiting small-scale dynamo action, and those sustaining the field on the large-scale (Brummell et al. 2001). It was presented that nonlinear gaseous flows that were responsible for the local dynamos were not directly connected to the large-scale dynamos. Thus there are gas motions that do not locally amplify the magnetic field but they could give the largescale dynamo and flows responsible for the local dynamos, resulting in a much smaller $\alpha$-effect (Brummell et al. 2001). For these reasons we decided to calculate the EMF coefficients as time-dependent quantities. They are evaluated within the limit of high microscopic conductivity.

\section{Basic equations and the dynamo coefficients}

The basic quantity in mean-field electrodynamics is the mean electromotive force (EMF)

$\mathcal{E}=\left\langle\boldsymbol{u}^{\prime} \times \boldsymbol{B}^{\prime}\right\rangle$,

whose curl determines the time evolution of the mean magnetic field $\langle\boldsymbol{B}\rangle$ :

$\frac{\partial\langle\boldsymbol{B}\rangle}{\partial t}=\operatorname{curl}(\mathcal{E}+\langle\boldsymbol{u}\rangle \times\langle\boldsymbol{B}\rangle)$,

with $\boldsymbol{u}$ and $\boldsymbol{B}$ being the velocity and the magnetic field induction, respectively. In these equations the notation $\langle F\rangle$ (where $F$ concerns $\boldsymbol{u}$ or $\boldsymbol{B}$ ) denotes quantities averaged by a procedure according to the Reynolds rules (e.g. Moffatt 1978) usually an ensemble average. $F^{\prime}$ represents fluctuations (see Otmianowska-Mazur et al. 1997). Instead of averaging over an ensemble one can also define mean values by the integration in space. In the present simulations, angular brackets denote averaging in planes parallel to the $X Y$ plane (the galactic plane) in order to get the $Z$-dependence of both quantities. This implies that the coefficients are constant in planes, however the derivation of non-isotropic magnetic EMF coefficients is also possible (Rogachevskii \& Kleeorin 2001; Kleeorin \& Rogachevskii 1999), but this is a separate issue.

From our model of Parker instability (see OtmianowskaMazur et al. 2003; Kowal et al. 2003a,b and Paper I), we obtained helical small-scale gas motions evolving in a rectangular parcel (in Cartesian coordinates). In the present study we estimate how strong an $\alpha$-effect we can get from our previous 
simulations. Thus we calculate simultaneously to our Parker instability computations the resultant components of both tensors $\hat{\alpha}$ and $\hat{\eta}$ as time-dependent quantities. We restrict ourselves to the computation of the turbulent EMF (Eq. (1)) in the highconductivity limit. Then the second-order correlation approximation yields the expression (Rädler 1980):

$$
\begin{aligned}
\mathcal{E}_{\mathrm{i}}(\boldsymbol{x}, t)= & \int_{0}^{\infty}\left(\hat{\alpha}_{i j}(\boldsymbol{x}, t, \tau)\left\langle\boldsymbol{B}_{j}\right\rangle(\boldsymbol{x}, t-\tau ; t)\right. \\
& \left.+\hat{\eta}_{i j k}(\boldsymbol{x}, t, \tau) \frac{\partial\left\langle\boldsymbol{B}_{j}(\boldsymbol{x}, t-\tau ; t)\right\rangle}{\partial x_{k}}\right) \mathrm{d} \tau
\end{aligned}
$$

with the dynamo coefficients $\hat{\alpha}_{i j}$ and $\hat{\eta}_{i j k}$, and $\boldsymbol{B}_{j}$ as the magnetic field components. Detailed expressions for the EMF are given by Krause \& Rädler (1980). We apply them for a Cartesian geometry where $y$ represents the azimuthal direction and $x$ the radial one. Both tensors are calculated from the same velocity field.

It is convenient to write locally Eq. (3) in the form of its components:

$$
\begin{aligned}
& \mathcal{E}_{x}=\alpha_{x x}\left\langle B_{x}\right\rangle+\alpha_{x y}\left\langle B_{y}\right\rangle+\eta_{T} \frac{\partial\left\langle B_{y}\right\rangle}{\partial z}-\eta_{T} \frac{\partial\left\langle B_{z}\right\rangle}{\partial y}, \\
& \mathcal{E}_{y}=\alpha_{y y}\left\langle B_{y}\right\rangle+\alpha_{y x}\left\langle B_{x}\right\rangle-\eta_{T} \frac{\partial\left\langle B_{x}\right\rangle}{\partial z}+\eta_{T} \frac{\partial\left\langle B_{z}\right\rangle}{\partial x}, \\
& \mathcal{E}_{z}=\alpha_{z z}\left\langle B_{z}\right\rangle-\eta_{T} \frac{\partial\left\langle B_{y}\right\rangle}{\partial x}+\eta_{T} \frac{\partial\left\langle B_{x}\right\rangle}{\partial y} .
\end{aligned}
$$

In our model we apply only the mean diffusion coefficient $\eta_{\mathrm{T}}$ defined by Eq. (7), due to the fact that we are mainly interested in components of the $\hat{\alpha}_{i j}$ tensor (see Eq. (5)) representing the amplification and the buoyancy mechanisms of the magnetic field in galactic disks.

For homogeneous and isotropic small-scale gas motions the $\alpha$-tensor has no non-diagonal elements; however as we wrote in Sect. 1 we analyze the model with a fully anisotropic velocity field and because of that we should check the strength of the buoyancy force by also computing the antisymmetric part of $\alpha_{y x}$. From Moffatt (1978) and Otmianowska-Mazur et al. (1997) it follows that

$$
\begin{aligned}
& \alpha_{x x}=\int_{0}^{\infty}\left\langle u_{y}(t) \frac{\partial u_{z}(t-\tau)}{\partial x}-u_{z}(t) \frac{\partial u_{y}(t-\tau)}{\partial x}\right\rangle \mathrm{d} \tau, \\
& \alpha_{y y}=\int_{0}^{\infty}\left\langle u_{z}(t) \frac{\partial u_{x}(t-\tau)}{\partial y}-u_{x}(t) \frac{\partial u_{z}(t-\tau)}{\partial y}\right\rangle \mathrm{d} \tau, \\
& \alpha_{x y}=\int_{0}^{\infty}\left\langle u_{y}(t) \frac{\partial u_{z}(t-\tau)}{\partial y}-u_{z}(t) \frac{\partial u_{y}(t-\tau)}{\partial y}\right\rangle \mathrm{d} \tau, \\
& \alpha_{y x}=\int_{0}^{\infty}\left\langle u_{z}(t) \frac{\partial u_{x}(t-\tau)}{\partial x}-u_{x}(t) \frac{\partial u_{z}(t-\tau)}{\partial x}\right\rangle \mathrm{d} \tau, \\
& \alpha_{z z}=\int_{0}^{\infty}\left\langle u_{x}(t) \frac{\partial u_{y}(t-\tau)}{\partial z}-u_{y}(t) \frac{\partial u_{x}(t-\tau)}{\partial z}\right\rangle \mathrm{d} \tau .
\end{aligned}
$$

Commonly one can separate the abovementioned antisymmetric part of $\alpha_{y x}$ as (e.g. Ossendrijver et al. 2002; Moffatt 1978):

$\alpha_{y x}^{A}=\frac{\left(\alpha_{y x}-\alpha_{x y}\right)}{2}$

The diffusion coefficient is calculated according to the following form:

$$
\begin{aligned}
\eta_{\mathrm{T}}= & \frac{1}{3}\left[\int_{0}^{\infty}\left\langle u_{x}(t) u_{x}(t-\tau)\right\rangle \mathrm{d} \tau+\int_{0}^{\infty}\left\langle u_{y}(t) u_{y}(t-\tau)\right\rangle \mathrm{d} \tau\right. \\
& \left.+\int_{0}^{\infty}\left\langle u_{z}(t) u_{z}(t-\tau)\right\rangle \mathrm{d} \tau\right]
\end{aligned}
$$

This equation allows also for negative values of the diffusion coefficients, an effect expected for the dynamo theory (see Parker 1979).

\section{The model of small-scale gas motion}

In the present numerical model we study the time evolution of the dynamo coefficients (Eq. (3)) induced by the Parker instability mechanism as presented in Paper I. In Paper I we analyzed the 3D evolution of the Parker instability in the presence of rigid rotation and fast magnetic reconnection (without shear) in a parcel of galactic gas situated in the vicinity of the Sun (Paper I; Kowal et al. 2003a; Otmianowska-Mazur et al. 2003). Our initial equilibrium state was an exponentially stratified disk as proposed by Parker (1966). The equilibrium state was characterized by uniform vertical gravity, an isothermal equation of state and a sound speed constant across the disk. The fixed ratio of the magnetic pressure to the gas pressure was given by $\alpha_{\text {dyn }}=p_{\text {mag }} / p_{\text {gas }}$. In a Cartesian reference frame with coordinates $x, y, z$ corresponding locally to the radial, azimuthal and vertical coordinates in the disk, the assumed initial magnetic field was purely azimuthal, $B_{0}=B_{0}(z) e_{y}$ and the dependence of equilibrium quantities on $z$ was

$\frac{p_{0}(z)}{p_{0}(0)}=\frac{n_{0}(z)}{n_{0}(0)}=\frac{B_{0}^{2}(z)}{B_{0}^{2}(0)}=\exp \left(\frac{-z}{H}\right)$

with the midplane particle density $n_{0}(0)=1 \mathrm{~cm}^{-3}$, the isothermal sound speed $c_{\mathrm{s}}=7 \mathrm{~km} \mathrm{~s}^{-1}$, vertical gravitational acceleration $g=-2 \times 10^{-9} \mathrm{~cm} \mathrm{~s}^{-2}, \alpha_{\text {dyn }}=1.0$ and a vertical scaleheight $H=\left(1+\alpha_{\text {dyn }}\right) c_{\mathrm{s}}^{2} /|g| \simeq 150$ pc. We used the angular velocity of the galactic rotation of the Solar orbit $\Omega=0.025 \mathrm{Myr}^{-1}$. The chosen value of $\alpha_{\mathrm{dyn}}=1.0$ corresponds to the magnetic field of $4.5 \mu \mathrm{G}$ at the galactic midplane (see Paper I for explanation). We used the concept of an anomalous resistivity which depended on the current density (Ugai 1992; Tanuma et al. 1999, 2001; Konz et al. 2000)

$\eta(j)=\eta_{2}\left(j^{2}-j_{\text {crit }}^{2}\right)^{1 / 2} \Theta\left(j^{2}-j_{\text {crit }}^{2}\right)$,

where $\eta_{2}$ was a constant coefficient, $j_{\text {crit }}$ denoted the critical current density above which the resistivity switches on and $\Theta$ was the Heaviside step function (see Paper I for explanation). With the help of the Zeus-3D code (Stone \& Norman 1992a,b) 


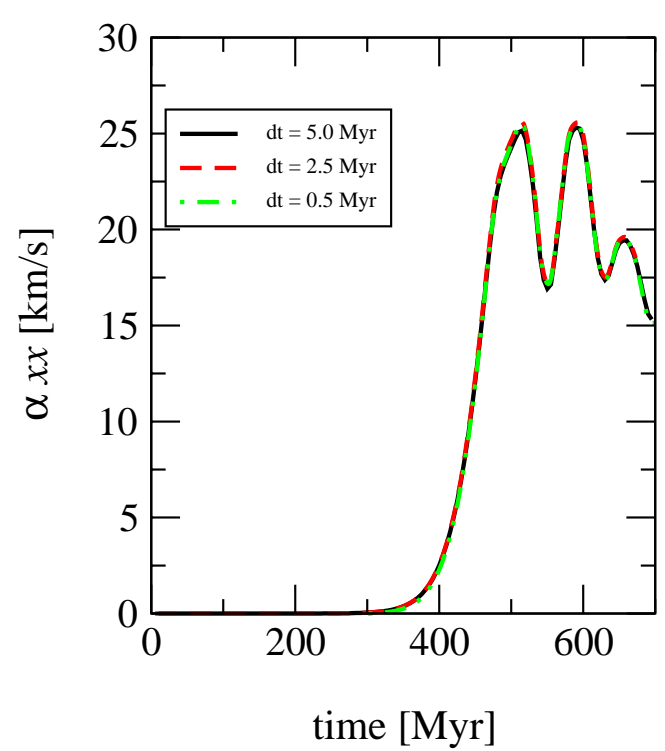

Fig. 1. The evolution of the dynamo coefficient $\alpha_{x x}$ for the model D1; the height above the galactic plane is $z=300 \mathrm{pc}$ for different time steps.

in Paper I we performed a series of more than 20 numerical simulations of the Parker instability applying different perturbations. The major simulations were done with the resolution of $120 \times 90 \times 240$ zones in the $x, y$ and $z$ directions, respectively. To check the effect of the finite grid resolution we repeated the same simulations with the resolution of $60 \times 90 \times 120$ grid cells; however, these simulations differed only in minor details compared to the high resolution cases. For all the simulations we applied the Cartesian computational domain of physical sizes of $L_{x}=600 \mathrm{pc}, L_{y}=1800 \mathrm{pc}$ and $L_{z}=600 \mathrm{pc}$.

In our present simulations, at every time step the resulting velocity field (from our Parker instability model) is used to calculate the EMF coefficients (Eqs. (5) and (7)). Due to the Coriolis force and the density stratification, the small-scale motions of gas possess a helical character, so we decided to compute both dynamo coefficients in two chosen experiments from 20 simulations presented in Paper I. In order to start the evolution of the Parker instability we assumed that at the beginning a multicomponent velocity perturbation in the form of waves occurs. The perturbations are given by the formula:

$$
\begin{aligned}
u_{z}(x, y, z)= & \sum_{i_{x}, i_{y}, i_{z}=1}^{n_{x}, n_{y}, n_{z}} \frac{v_{0}}{n_{x} n_{y} n_{z}} \cos \left(\frac{2 \pi i_{x} x}{L_{x}}+\phi_{i_{x}}\right) \\
& \times \cos \left(\frac{2 \pi i_{y} y}{L_{y}}+\phi_{i_{y}}\right) \sin \left(\frac{\pi i_{z} z}{H}+\phi_{i_{z}}\right)
\end{aligned}
$$

where $n_{x}, n_{y}$ and $n_{z}$ are the numbers of harmonic components in the $x, y$ and $z$ direction, $\phi_{i_{x}}, \phi_{i_{y}}$ and $\phi_{i_{z}}$ are the random phases. The limitation of the perturbations to $z \leq H$ is motivated by the assumption that the stellar activity in the disk (e.g. supernova explosions, stellar winds or cosmic rays) initiates the instability.

\section{Numerical simulations}

In order to estimate the dependence of the dynamo coefficients computed on the grid size (Eqs. (5) and (7)) we calculated two experiments: B1 with the smallest $\left(n_{x}=3, n_{y}=3, n_{z}=1\right)$ and D1 with the highest $\left(n_{x}=10, n_{y}=5, n_{z}=5\right)$ numbers of harmonic components of the velocity perturbations (see Paper I) using the time step of 5.0 Myr for both experiments. To check the accuracy of the integration procedure, the experiment D1 was recalculated for two smaller time steps: $2.5 \mathrm{Myr}$ and 0.5 Myr. We average both dynamo coefficients over all planes parallel to the $X Y$ plane at the chosen height $z$, because we would like to compare these quantities with magnetic field components averaged in the same manner in Paper I (Fig. 8). We analyze the time evolution of the turbulent diffusivity and the $\hat{\alpha}$ tensors as well as their time averaged values $E(X)$ and rms time variations $\sigma(X), X$ being the components of $\alpha_{i j}$ or $\eta_{\mathrm{T}}$. The standard deviation is computed in order to give information how big the fluctuations are in comparison with the timeaveraged value of a given quantity for the models B1 (Table 1) and D1 (Table 2). In order to check how the magnetic field strength influences the dynamo coefficients we recalculated the model B1 with 2 times lower initial magnetic field for the low resolution case (the model $\mathrm{B} 2$ ). In such case the ratio of magnetic to gaseous pressure is $1 / 4$ ( 1 in B1 and D1 experiments, see Paper I).

\section{Results}

The dependence of our simulations on the adopted time step is presented in Fig. 1 as the time evolution of $\alpha_{x x}$ resulting from the model D1 for three different time steps: $5 \mathrm{Myr}, 2.5 \mathrm{Myr}$ and $0.5 \mathrm{Myr}$, computed at the height of $300 \mathrm{pc}$ above the galactic plane. The three curves obtained have very similar shapes indicating that the process of integration in our code is not dependent on the time step and we can compute models for the largest time step of $5 \mathrm{Myr}$ without losing accuracy.

Figure 2 shows the time evolution of the coefficient $\alpha_{x x}$ obtained from the experiments B1 (left) and D1 (right) at the cho$\operatorname{sen} z$ above the galactic plane with the high $(120 \times 90 \times 240)$ and low $(60 \times 90 \times 120)$ grid resolution. The evolution presented starts from $250 \mathrm{Myr}$ because before that time the value of all coefficients is about zero. The dotted lines are for the low resolution case, the solid lines are for the high one. In both experiments there are no big differences between the high and low resolution models. This means that calculations with both resolutions give similar results for the dynamo coefficients. For this reason in Figs. 3-6 we present results for the high resolution computations, while the low resolution is applied for the model B2 (with an initial magnetic field two times weaker than for the run B2 see Figs. 7-9).

The time evolution of coefficients $\alpha_{y y}$ starting from 250 Myr for the high resolution models B1 and D1 are shown in Fig. 3 left and right respectively, again at three chosen heights above the galactic plane. For the model B1 the coefficient $\alpha_{x x}$ (see Fig. 2 left) reaches its maximum value of about $80 \mathrm{~km} \mathrm{~s}^{-1}$ at the time step of about $425 \mathrm{Myr}$ at the height of $700 \mathrm{pc}$. Two cuts at lower $z$ show much smaller values. Experiment 

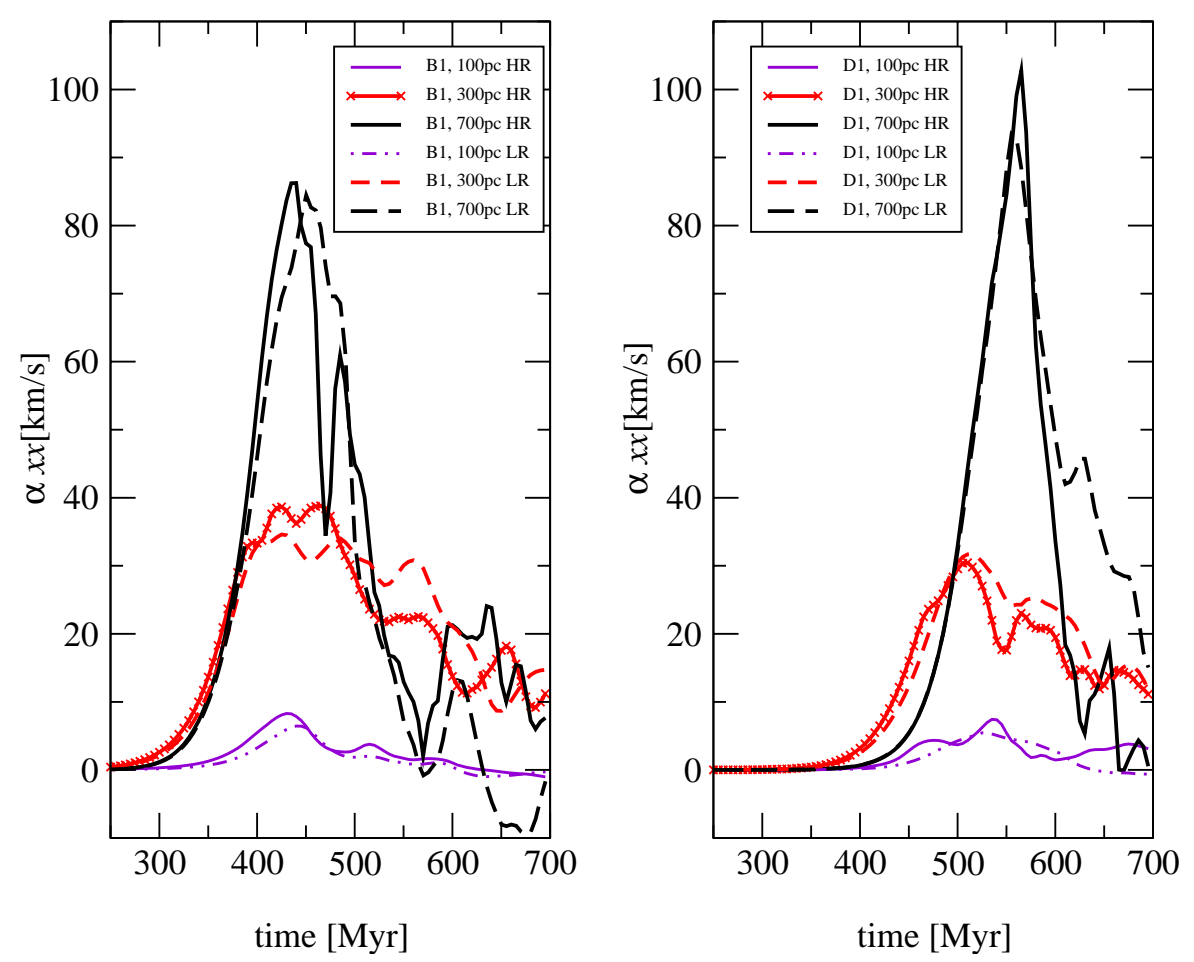

Fig. 2. The time evolution of the dynamo coefficient $\alpha_{x x}$ for the models D1 (right) and B1 (left) at three chosen heights above the disk plane for the high (HR, solid lines) and low (LR, dotted lines) resolution models.
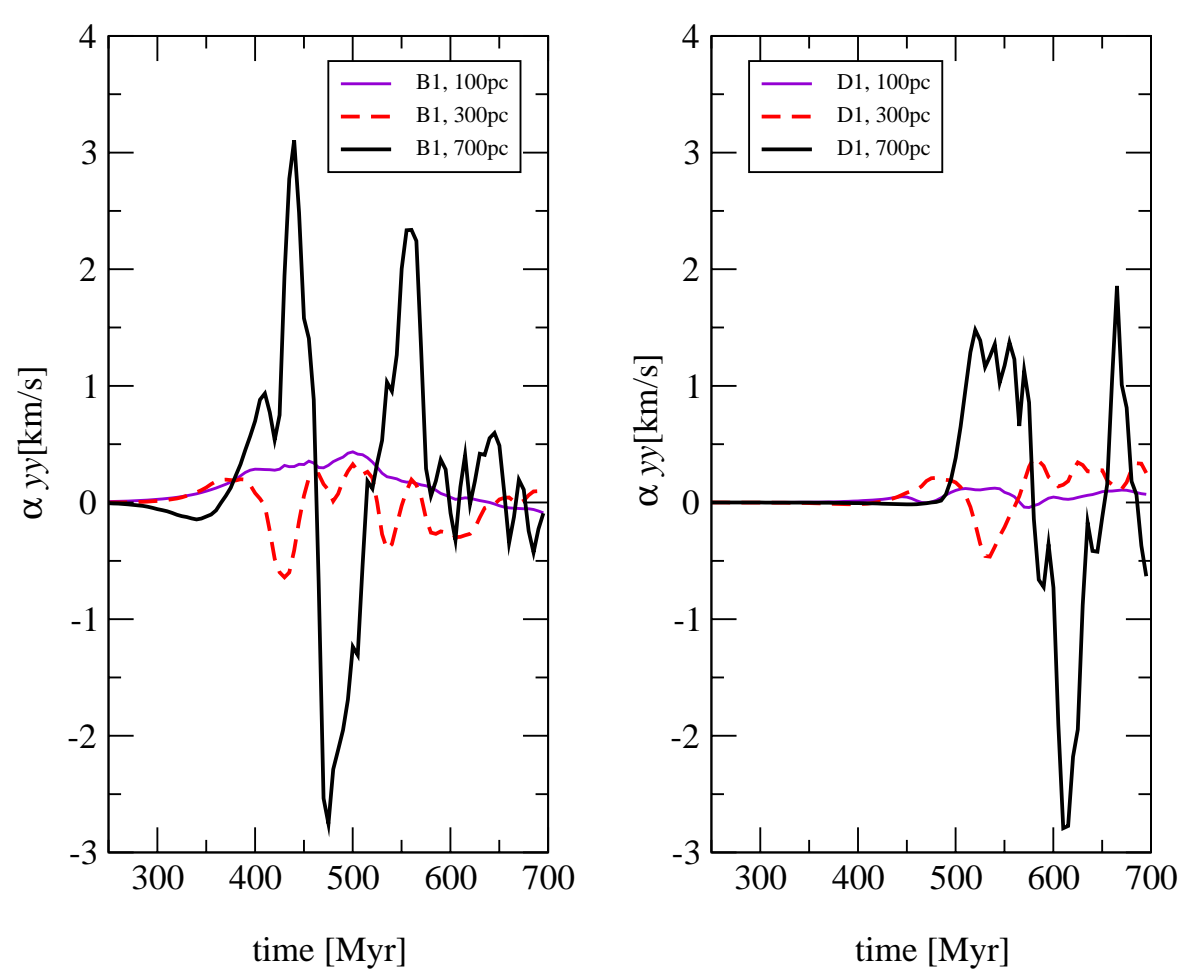

Fig. 3. The time evolution of the dynamo coefficients $\alpha_{y y}$ for the models B1 (left) and D1 (right) at three chosen heights above the disk plane for the high resolution models. The curves are described in the insets.

D1 yields a maximum value of about $100 \mathrm{~km} \mathrm{~s}^{-1}$ (see Fig. 2, right). The position of this maximum is shifted significantly in time to about $550 \mathrm{Myr}$, thus later in comparison with B1. The component $\alpha_{x x}$ for two cuts at lower $z$ again has smaller values (Fig. 2). The time changes of the second coefficient $\alpha_{y y}$ looks very different (Fig. 3). The growth of this component is not so efficient, probably due to the small value of the random velocity in the $X$ direction (see Discussion). It reaches its maximum of about $3 \mathrm{~km} \mathrm{~s}^{-1}$ at $450 \mathrm{Myr}$ for the case B1 at $z$ of $700 \mathrm{pc}$ above the galactic plane (see Fig. 3 left). During the 

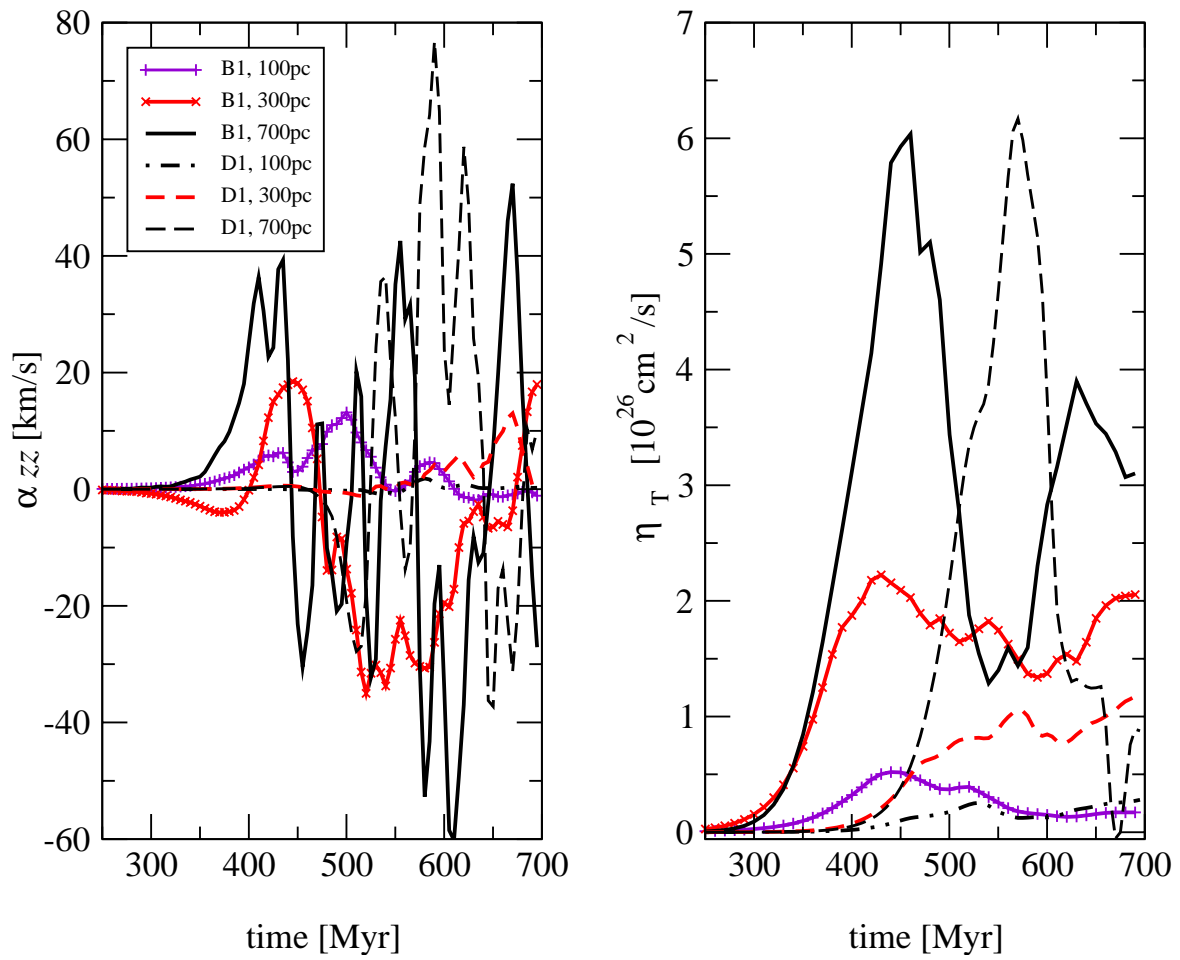

Fig. 4. The time evolution of the dynamo coefficient $\alpha_{z z}$ (left) and the diffusion coefficient $\eta_{\mathrm{T}}$ (right) for both models at three chosen heights above the disk plane for the high resolution models. The curves are described in the inset.

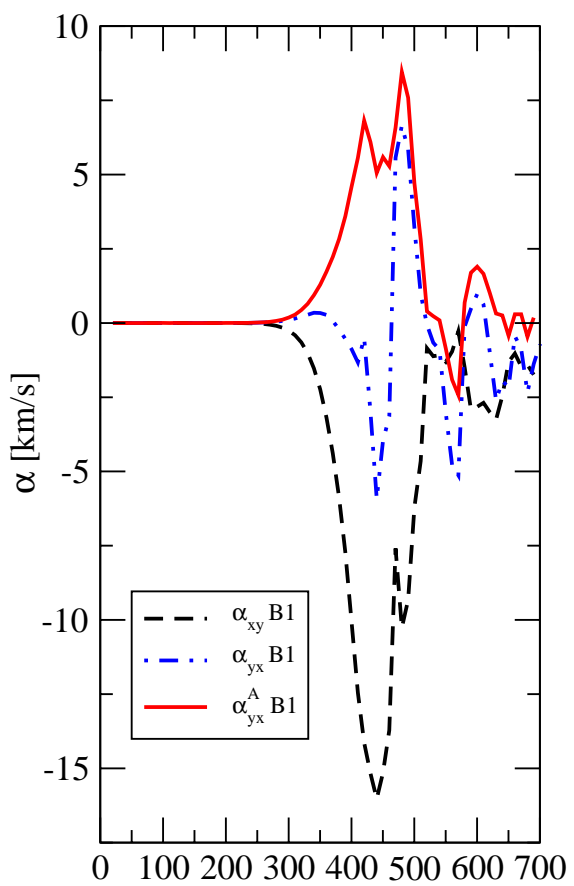

time $[\mathrm{Myr}]$

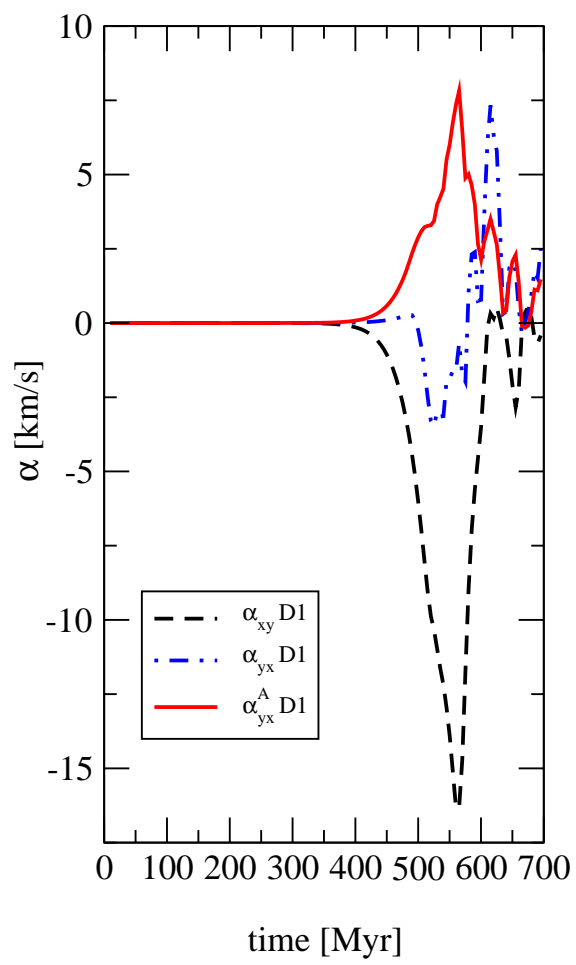

Fig. 5. The time evolution of three dynamo coefficients: $\alpha_{y x}^{A}$ (solid lines), $\alpha_{y x}$ (dashed-dotted lines) and $\alpha_{x y}$ (dashed lines) for the model B1 (the left panel) and D1 (the right panel) at $z=700 \mathrm{pc}$. The curves are described in the insets.

next 50 Myr it oscillates rapidly around zero with an amplitude of $3 \mathrm{~km} \mathrm{~s}^{-1}$. Later on, the amplitude decreases while the oscillations persist until the end of the evolution. The rest of the curves at lower $z$ behave similarly, however with a smaller amplitude of variations. The same coefficient for the model D1 has an even smaller maximum value than in the experiment B1 (Fig. 3 right).

Figure 4 left panel shows the time-evolution of the coefficient $\alpha_{z z}$, while the right panel presents it for $\eta_{\mathrm{T}}$. The presented evolution starts again from 250 Myr. In the classical 

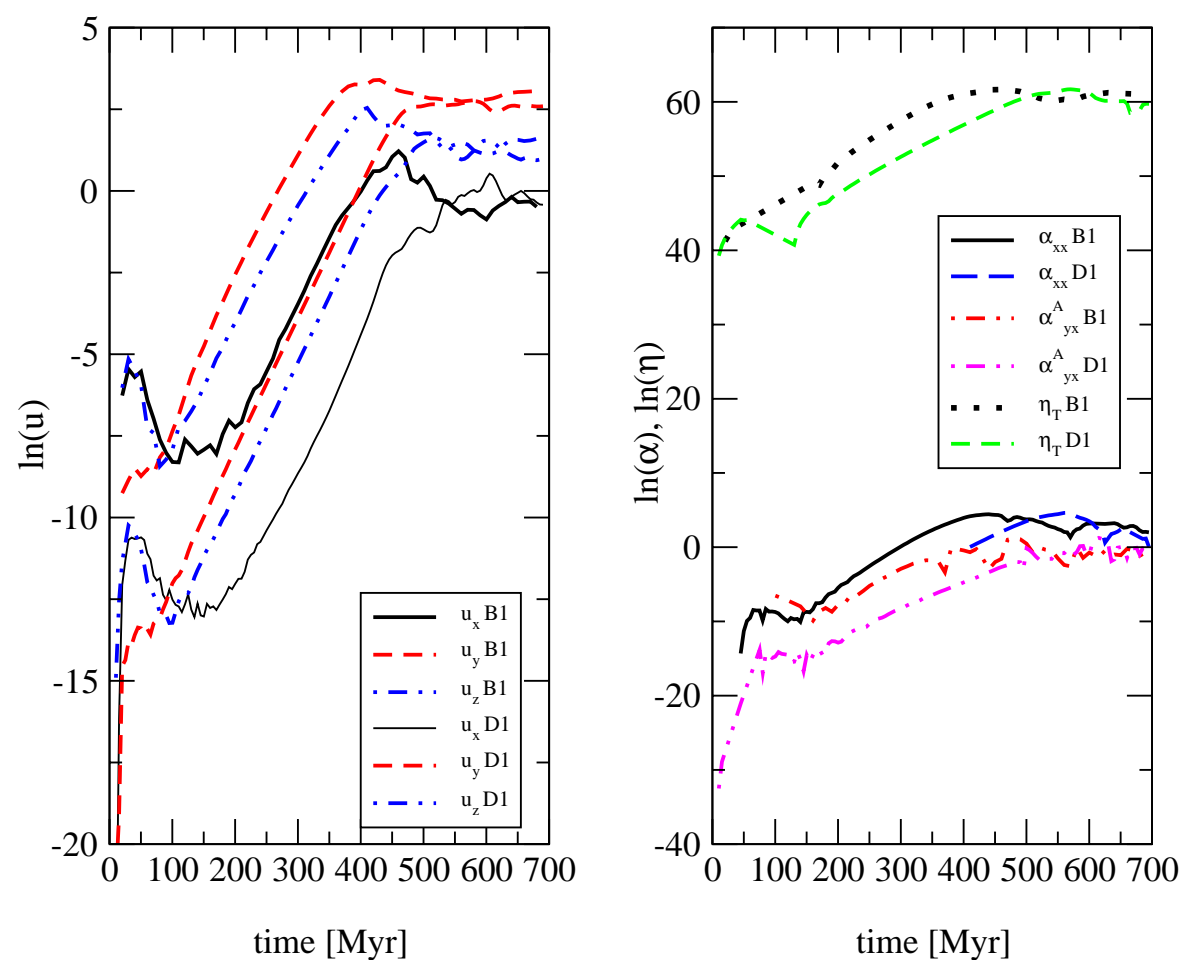

Fig. 6. The time evolution of the natural logarithm of the mean square velocity (left) and the chosen dynamo coefficients (right) for both models at $700 \mathrm{pc}$ height above the galactic plane for the high resolution models. The curves are described in the insets.
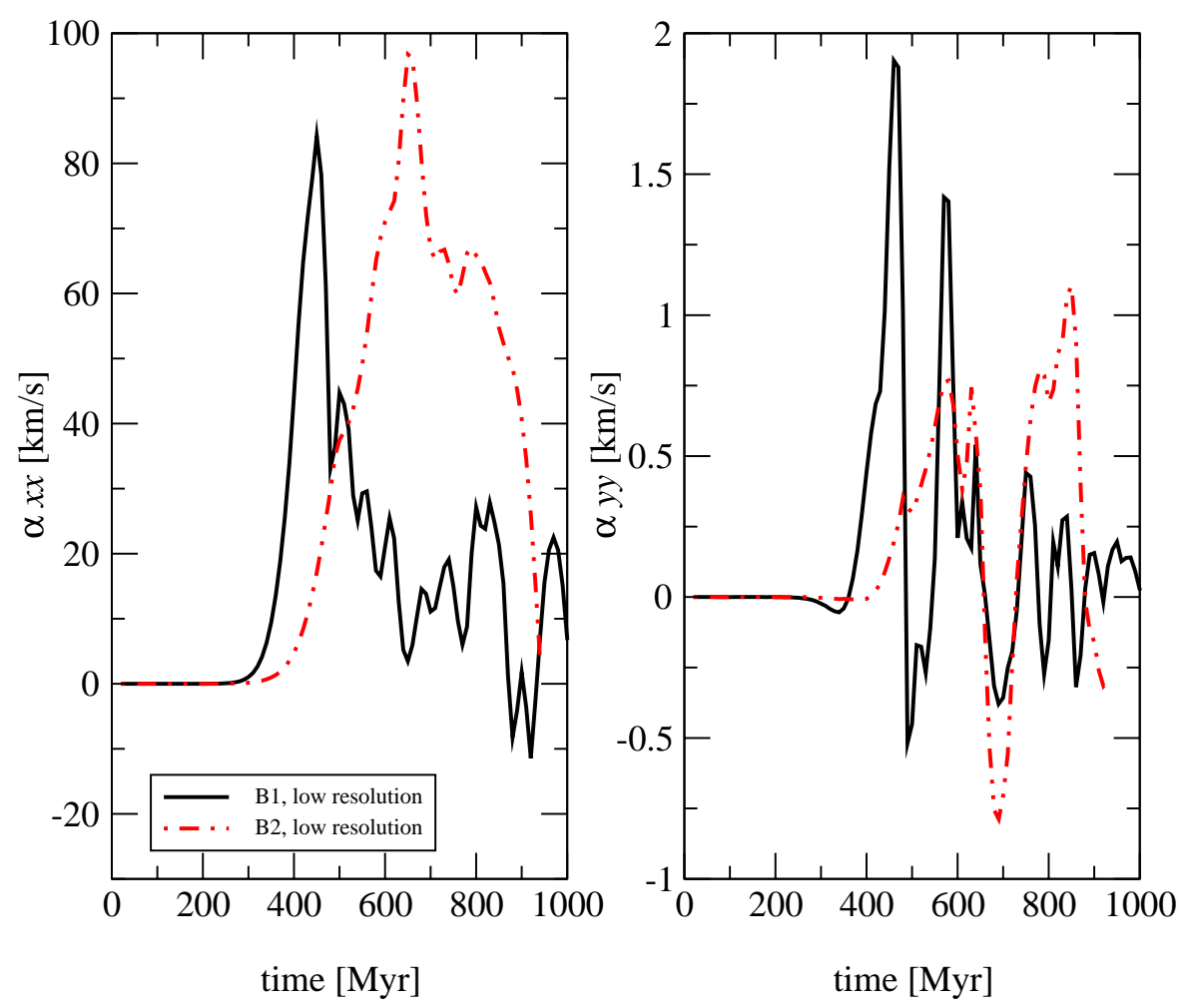

Fig. 7. The time evolution of the dynamo coefficient $\alpha_{x x}$ (left) and $\alpha_{y y}$ (right) for the model B1 (solid lines) and B2 (dashed-dotted lines) at $z=700$ pc. The experiment B1 has two times stronger the initial magnetic field than B2.

dynamo theory (Ruzmaikin et al. 1988), $\alpha_{z z}$ should be negative above the galactic plane due to the Coriolis force (see Discussion). The evolution of our modeled coefficient is more complex. It changes its sign from plus to minus with extreme values at the height of $700 \mathrm{pc}$ of $+80 \mathrm{~km} \mathrm{~s}^{-1}$ and $-60 \mathrm{~km} \mathrm{~s}^{-1}$ for the models D1 and B1, respectively. The coefficient of magnetic diffusion $\eta_{\mathrm{T}}$ presented in Fig. 4 (right panel) shows a similar evolution to $\alpha_{x x}$. B1 yields a maximum value of about 

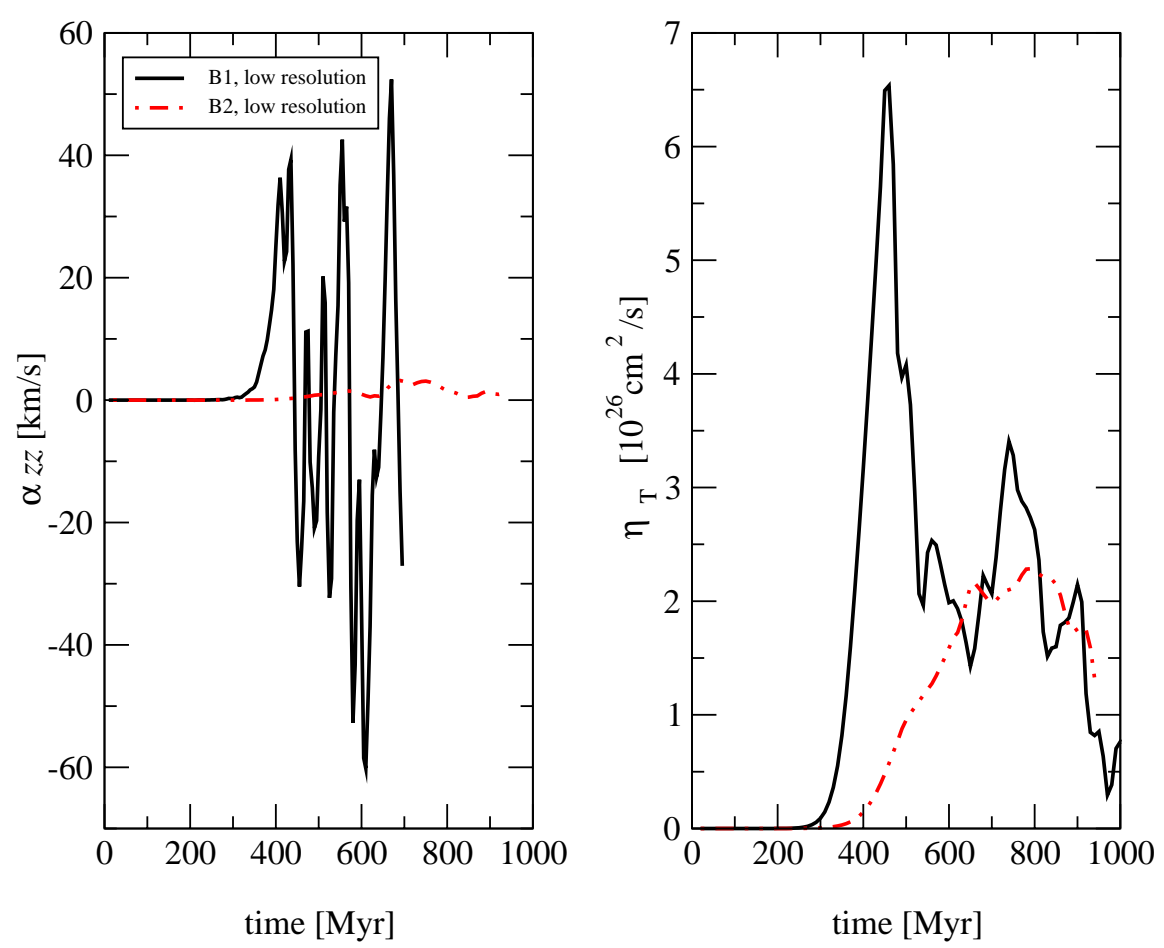

Fig. 8. The time evolution of the dynamo coefficient $\alpha_{z z}$ (left) and the diffusion coefficient $\eta_{\mathrm{T}}$ (right) for the model B1 (solid lines) and B2 (dashed-dotted lines) at $z=700 \mathrm{pc}$. The experiment B1 has a two times stronger initial magnetic field than B2.
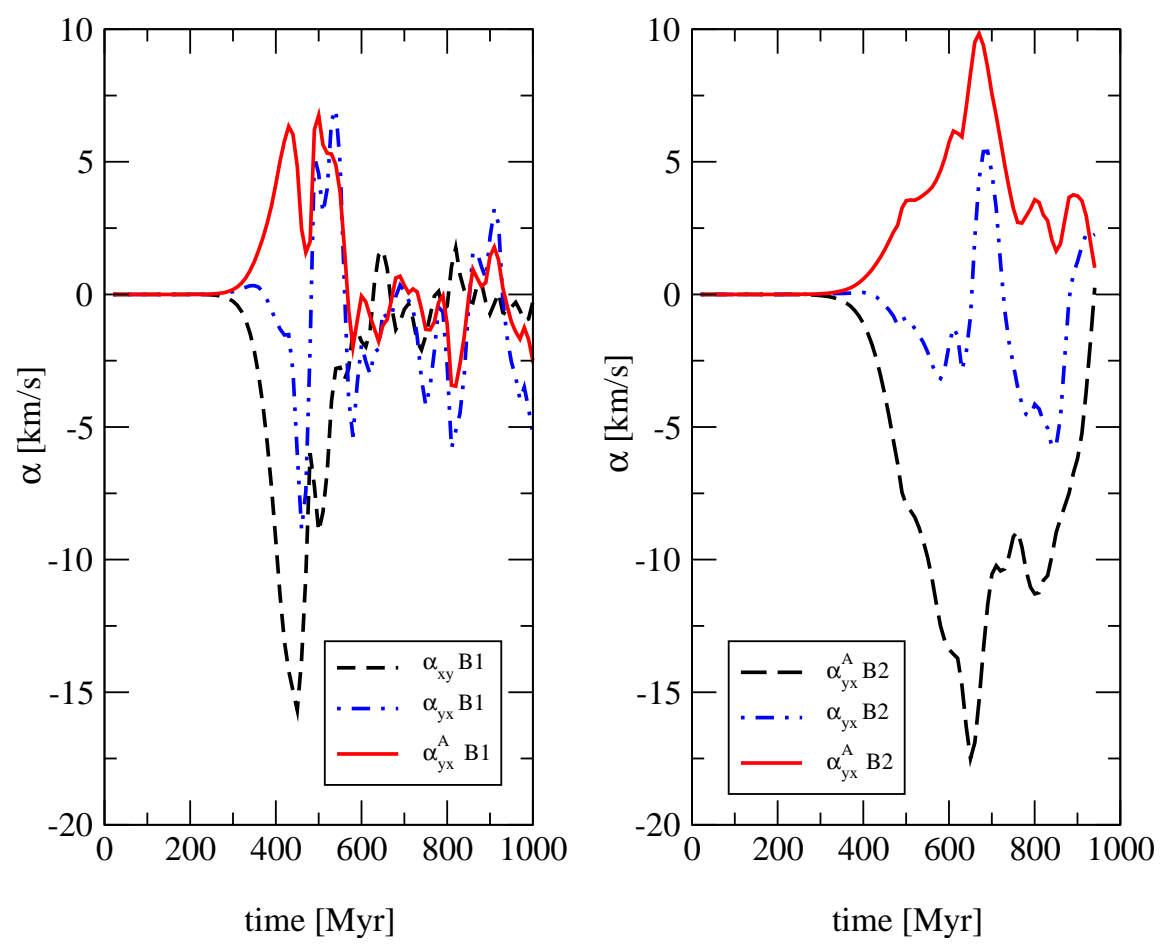

Fig. 9. The time evolution of three dynamo coefficients: $\alpha_{y x}^{A}$ (solid lines), $\alpha_{y x}$ (dashed-dotted lines) and $\alpha_{x y}$ (dashed lines) for the model B1 (the left panel) and B2 (the right panel) at $z=700 \mathrm{pc}$. The experiment B1 has a two times stronger initial magnetic field than B2.

$6 \times 10^{26} \mathrm{~cm}^{2} \mathrm{~s}^{-1}$ at $450 \mathrm{Myr}$. The peaks in D1 are shifted to a later time of about $575 \mathrm{Myr}$ with a similar maximum value of $6.0 \times 10^{26} \mathrm{~cm}^{2} \mathrm{~s}^{-1}$.

The time evolution of the antisymmetric part of $\alpha_{i j}$ is presented in Fig. 5 for the high resolution models B1 (left panel) and D1 (right panel). Our simulations show that small-scale motions of gas evolving in the Parker instability process are highly anisotropic: the tensor component $\alpha_{y x} \neq$ $-\alpha_{x y}$. In both models $\alpha_{x y}$ (dashed lines) has a much higher extremum (about $-15 \mathrm{~km} \mathrm{~s}^{-1}$ ) than $\alpha_{y x}$ (dashed-dot lines). The component $\alpha_{y x}$ for the model B1 (left panel) has in the beginning a slightly negative value (maximum about $-5 \mathrm{~km} \mathrm{~s}^{-1}$ ) then 
Table 1. The time-averaged values $E(X)$ and standard deviations $\sigma(X)$ of the dynamo tensor components for B1: the height is in pc, $\alpha$ in $\mathrm{km} \mathrm{s}^{-1}$ and $\eta$ is in $\mathrm{cm}^{2} \mathrm{~s}^{-1}$.

\begin{tabular}{ccccccccccc}
\hline \hline Height & $E\left(\alpha_{x x}\right)$ & $\sigma\left(\alpha_{x x}\right)$ & $E\left(\alpha_{y y}\right)$ & $\sigma\left(\alpha_{y y}\right)$ & $E\left(\alpha_{z z}\right)$ & $\sigma\left(\alpha_{z z}\right)$ & $E\left(\alpha_{y x}^{A}\right)$ & $\sigma\left(\alpha_{y x}\right)$ & $E\left(\eta_{\mathrm{T}}\right)$ & $\sigma\left(\eta_{\mathrm{T}}\right)$ \\
\hline 700 & 16.76 & 23.56 & 0.14 & 0.84 & 0.48 & 18.99 & 1.27 & 2.30 & $16.8 \times 10^{25}$ & $19.3 \times 10^{25}$ \\
300 & 12.71 & 13.44 & -0.01 & 0.17 & -3.92 & 11.87 & 0.85 & 0.89 & $9.3 \times 10^{25}$ & $8.9 \times 10^{25}$ \\
100 & 1.36 & 2.77 & 0.10 & 0.14 & 1.62 & 3.12 & -0.02 & 0.07 & $1.2 \times 10^{25}$ & $1.3 \times 10^{25}$ \\
\hline
\end{tabular}

Table 2. The time-averaged values $E(X)$ and standard deviations $\sigma(X)$ of the dynamo tensor components for the experiment D1: the height is in pc, $\alpha$ in $\mathrm{km} \mathrm{s}^{-1}$ and $\eta$ is in $\mathrm{cm}^{2} \mathrm{~s}^{-1}$.

\begin{tabular}{ccccccccccc}
\hline \hline Height & $E\left(\alpha_{x x}\right)$ & $\sigma\left(\alpha_{x x}\right)$ & $E\left(\alpha_{y y}\right)$ & $\sigma\left(\alpha_{y y}\right)$ & $E\left(\alpha_{z z}\right)$ & $\sigma\left(\alpha_{z z}\right)$ & $E\left(\alpha_{y x}^{A}\right)$ & $\sigma\left(\alpha_{y x}\right)$ & $E\left(\eta_{\mathrm{T}}\right)$ & $\sigma\left(\eta_{\mathrm{T}}\right)$ \\
\hline 700 & 12.38 & 24.45 & -0.07 & 0.15 & 2.03 & 16.37 & 1.02 & 1.78 & $8.9 \times 10^{25}$ & $15.4 \times 10^{25}$ \\
300 & 7.77 & 9.82 & 0.02 & 0.14 & 0.61 & 1.80 & 0.26 & 0.38 & $3.1 \times 10^{25}$ & $4.0 \times 10^{25}$ \\
100 & 1.40 & 1.93 & 0.01 & 0.14 & -0.10 & 0.32 & 0.01 & 0.06 & $0.6 \times 10^{25}$ & $0.8 \times 10^{25}$ \\
\hline
\end{tabular}

positive values (about $+8 \mathrm{~km} \mathrm{~s}^{-1}$ as the maximum value) oscillating to the end of simulations with decreasing amplitude. The component $\alpha_{y x}$ for the run D1 shows a similar character for time evolution (Fig. 5, right panel), however the amplitude of variations is smaller than in the previous simulation B1. The resulting antisymmetric part $\alpha_{y x}^{A}$ defined by Eq. (6) (solid lines) in both presented experiments (Fig. 5 left and right panels) attains a maximum of about $8 \mathrm{~km} \mathrm{~s}^{-1}$, showing oscillations with a smaller amplitude until the end of the evolution.

For general comparisons we present in Fig. 6, the left panel, the natural logarithm of the mean square velocities $\left(<u_{x}^{2}>\right.$, $<u_{y}^{2}>$ and $<u_{z}^{2}>$ ) averaged in space at a height of $700 \mathrm{pc}$. From these graphs we can easily get the saturation time of the modeled process, when the evolution of the Parker instability stops. The saturation time for small-scale gas motions in the model B1, assuming a low number of harmonic components of the velocity perturbations, is reached at about $450 \mathrm{Myr}$, 100 Myr earlier than for the run D1. We can see that the velocity component $u_{x}$ (solid lines) has the significantly smaller value of $3 \mathrm{~km} \mathrm{~s}^{-1}$, lower than the two other components $u_{y}$ and $u_{z}$, reaching $15 \mathrm{~km} \mathrm{~s}^{-1}$ and $5 \mathrm{~km} \mathrm{~s}^{-1}$ as the maximum, respectively. This fact is important in the evolution of $\alpha$ components (see Discussion). We compare three dynamo coefficients: $\ln \left(\alpha_{x x}\right), \ln \left(\alpha_{y x}^{A}\right)$ and $\ln \left(\eta_{\mathrm{T}}\right)$ in the same figure, the right panel. The rest of the coefficients strongly oscillate between positive and negative values and it is not possible to take their logarithm. As shown in the figure the presented coefficients have the same time scales of the growth as the velocities: $450 \mathrm{Myr}$ for the model B1 and 550 Myr for D1.

Tables 1 and 2 show the mean values and the standard deviations of the chosen quantities: $\alpha_{x x}, \alpha_{y y}, \alpha_{z z}, \alpha_{y x}^{A}$ and $\eta_{\mathrm{T}}$ averaged over the whole time period, for the runs B1 and D1, respectively. The calculations with high resolution are presented. The experiment with a smaller number of harmonic components of the velocity perturbations B1 has (in most cases) higher mean values of almost all dynamo components. For $\alpha_{x x}$ we have the mean value of about $16 \mathrm{~km} \mathrm{~s}^{-1}$ at $z=700 \mathrm{pc}$. The model D1 yields the mean $\alpha_{x x}$ of about $12 \mathrm{~km} \mathrm{~s}^{-1}$. Both runs give the standard deviation almost twice as large as the mean values of $\alpha_{x x}$. The mean values and standard deviations are smaller for cuts at lower $z$. The positive values of $\alpha_{x x}$ are in agreement with the expectations of helically twisted turbulences (due to the Coriolis force, see Discussion). The mean values of $\alpha_{y y}$ and $\alpha_{z z}$ are similar and very small. We note, however, that while $\alpha_{z z}$ oscillates strongly in time between $+80 \mathrm{~km} \mathrm{~s}^{-1}$ and $-60 \mathrm{~km} \mathrm{~s}^{-1}$ (note the high $\sigma$ in Table 1), $\alpha_{y y}$ stays small $\left( \pm 3 \mathrm{~km} \mathrm{~s}^{-1}\right)$ over the whole evolutionary time (see text for the model description). Fluctuations of $\alpha_{z z}$ in both models result in several $\mathrm{km} \mathrm{s}^{-1}$ as the standard deviation. The mean values of $\alpha_{y x}^{A}$ are about $1.02 \mathrm{~km} \mathrm{~s}^{-1}$ and $1.27 \mathrm{~km} \mathrm{~s}^{-1}$ for models D1 and B1, respectively at $z=700 \mathrm{pc}$. Their standard deviations are about 1.5 times higher than the mean values. The mean values of that component at two lower cuts are significantly smaller. For $\eta_{\mathrm{T}}$ the model B1 gives the mean value about $17 \times 10^{25} \mathrm{~cm}^{2} \mathrm{~s}^{-1}$, which is almost two times higher than in the model D1 with value of about $9 \times 10^{25} \mathrm{~cm}^{2} \mathrm{~s}^{-1}$ at $700 \mathrm{pc}$. The model B1 and D1 yield their standard deviations 1.5 and 2 times higher than their mean value, respectively (both models at $z=700 \mathrm{pc}$ ). The cuts at lower $z$ give again smaller values of the averaged quantities and the standard deviations.

In order to check how our process depends on the initial magnetic field strength we make additional simulations, B2, with the initial component $B_{y}$ two times lower than its value in the computations B1 and D1. The multicomponent velocity perturbations are similar to the experiment B1, so the modeled quantities can be compared with results of this model but with the low resolution case. Due to the weakness of the initial magnetic field, the Parker instability in the run B2 evolves significantly slower, however in all our experiments (B1, D1 and B2) the initial magnetic field pressure is in an analytical equilibrium with the gas pressure and the gravitational potential (see Paper I). In Figs. 7-9 for simplicity we present only quantities averaged in space at $z=700 \mathrm{pc}$.

Figure 7 presents the time evolution of two dynamo coefficients: $\alpha_{x x}$ (the left panel) and $\alpha_{y y}$ (the right panel) for the model B1 (solid lines) and B2 (dashed-dotted lines). The experiment B2 yields the maximum of $\alpha_{x x}$ about 1.2 times higher than the extremum for the model B1. It is shifted $200 \mathrm{Myr}$ later. The coefficient $\alpha_{y y}$ in the simulations B2 (dashed-dotted line) also shows the highest peak shifted to 800 Myr, but its 
maximum value is about two times smaller than in the model B1 (solid line). The situation is similar for $\alpha_{z z}$. The model B2 (dashed-dotted line) with a two times lower input magnetic fields shows 10 times smaller oscillations than the run B1 (see Fig. 8, the left panel, solid line). The situation for $\eta_{\mathrm{T}}$ (Fig. 8, the right panel) is also different to our previous simulations B1. The diffusion coefficient for our new experiment B2 (dashed-dotted line) shows only one peak reaching the value of about $2.4 \times 10^{26} \mathrm{~cm}^{2} \mathrm{~s}^{-1}$ at $800 \mathrm{Myr}$. This value is slightly smaller than the second maximum of $\eta_{\mathrm{T}}$ for the model B1 (solid line).

Figure 9 compares the time evolution of the antisymmetric part of the $\alpha$-effect: the components $\alpha_{x y}$ (dashed lines), $\alpha_{y x}$ (dashed-dotted lines) and combined $\alpha_{y x}^{A}$ (according to Eq. (6), solid lines) for cases of the strong (B1) and weak (B2) initial field. The model B2 is presented in the right panel of the figure, the run $\mathrm{B} 1$ in the left one. All presented quantities from the new simulations B2 have the maxima shifted to $650 \mathrm{Myr}$ in comparison with the simulations B1. The peaks of $\alpha_{x y}$ is 1.2 times higher than the equivalent maximum for the experiment B1, while $\alpha_{y x}^{A}$ is about 1.4 times higher than in the other model. These differences are absent for $\alpha_{y x}$ which has only a slightly smaller amplitude of oscillations.

\section{Discussion}

Our model shows that both coefficients $\alpha_{x x}$ and $\alpha_{z z}$ possess quite big peaks (positive or negative), much higher than in models assuming the quenching of the magnetic field by turbulent motion (Ziegler 1996). The time-averaged value of the first coefficient is also high with a mean value of about $16 \mathrm{~km} \mathrm{~s}^{-1}$. Ferrière in her papers computed the value of four dynamo coefficients, similar to our present study. Her semi-analytically obtained quantities averaged in time and space have a maximum of $6 \mathrm{~km} \mathrm{~s}^{-1}$ for $\alpha_{r}\left(\alpha_{x x}\right.$ in our case) and $2 \mathrm{~km} \mathrm{~s}^{-1}$ for $\alpha_{z z}$. The calculations were made without any assumption of the magnetic field quenching. The full 3D MHD simulations with the magnetic field response to the gas flows were done by Ziegler (1996). Due to the estimation method used in his model (the dynamo coefficients were calculated from EMF), values of only three dynamo components were computed. The peak value of $\alpha_{z z}$ was very small in comparison with our results, with the maximum of about $-2 \mathrm{~m} \mathrm{~s}^{-1}$. The negative value of that component in the Ziegler (1996) simulations was in agreement with the classical dynamo theory. The high value of $\alpha_{x x}$ in our model allows for direct amplification of the $B_{y}\left(B_{\phi}\right)$ magnetic field component according to Eq. (2) and could yield a so-called $\alpha^{2} \omega$-dynamo (see also Ziegler 1996; Moffatt 1978). Our $\alpha_{z z}$ coefficient changes sign from plus to minus resulting in quite low mean values, between $0.48 \mathrm{~km} \mathrm{~s}^{-1}$ (B1) and $2 \mathrm{~km} \mathrm{~s}^{-1}$ (D1) with $\sigma$ of the order of $20 \mathrm{~km} \mathrm{~s}^{-1}$.

The component $\alpha_{y y}$ in our present study has a much smaller mean value (e.g. $0.14 \mathrm{~km} \mathrm{~s}^{-1}$ for B1) than $2 \mathrm{~km} \mathrm{~s}^{-1}$ obtained by Ferrière (1998). On the other hand, our $\alpha_{y y}$ is much higher than this coefficient calculated by Ziegler (1996). His model yields only 2-6 $\mathrm{m} \mathrm{s}^{-1}$. Brandenburg \& Donner (1997) obtained also rather small value of this component, about $0.1 \mathrm{~m} \mathrm{~s}^{-1}$ or even smaller, recalculating their values to galactic units.
Additionally our $\alpha_{y y}$ changes sign over a time scale of $100 \mathrm{Myr}$. The component $\alpha_{y y}\left(\alpha_{\phi}\right)$ is essential for the $\alpha \omega$-dynamo working in galaxies, producing the radial component of the magnetic field. This component gives rise then to the azimuthal field due to the differential rotation of the galactic disk. Our resulting $\alpha_{y y}$ is certainly too small to build effective dynamo action in the galactic disk. Such a value of $\alpha_{y y}$ (estimations from the classical dynamo give a value of $1 \mathrm{~km} \mathrm{~s}^{-1}$, Ruzmaikin et al. 1988) is caused by the low value of the radial velocity $u_{x}$ (see Fig. 6, left), which is much smaller than the other velocity components (see Kowal et al. 2003a). This velocity is responsible for $\alpha_{y y}$ (see Eq. (5)). The rest of the dynamo components also have terms without $u_{x}$. This small velocity value is caused by the character of gas motions in the Parker instability, which allows for the gas flow along magnetic lines of force - along the $Y$ and $Z$ direction in our case. Across the magnetic field lines the gas flow is limited (see Kowal et al. 2003a). This means that $\alpha_{y y}$ strongly depends on the geometry of the initial magnetic field.

The next important problem is the compatibility of the obtained $\hat{\alpha}$ coefficients with the results of Paper I. Our simulations made in Paper I yielded a quite significant radial component $B_{x}$ after 500 Myr of MHD evolution. This means that this component can be converted by the shear to the azimuthal one, amplifying the magnetic energy (yielding of small-scale dynamo action). In Paper I, due to the lack of the shearing process we have not obtained the amplification of the magnetic field energy so far. We expect we will detect it when we incorporate this process into our model. The very crude estimation of the value of the effective local $\alpha_{y y}$, which is necessary to get a significant radial component and is computed from the dynamo equation, gives the value of about $6 \mathrm{~km} \mathrm{~s}^{-1}$ at $z=0 \mathrm{pc}$ (after $500 \mathrm{Myr}$ ). We apply here $10^{26} \mathrm{~cm}^{2} \mathrm{~s}^{-1}$ as the mean value of the diffusion coefficient. The large-scale value (computed from Eq. (5) and averaged over time) of $\alpha_{y y}$ from the present calculations at lower $z$ is much smaller (see Table 1). At $z=100 \mathrm{pc}$ the local $\alpha_{y y}$ is about $-3 \mathrm{~km} \mathrm{~s}^{-1}$, at $z=200 \mathrm{pc}: 1.5 \mathrm{~km} \mathrm{~s}^{-1}$ and above $400 \mathrm{pc}$ this quantity decreases to about $0 \mathrm{~km} \mathrm{~s}^{-1}$. Thus, the so-called effective local $\alpha_{y y}$ has its maximum near the bottom of the cube opposite to the large-scale $\alpha_{y y}$ which has a maximum value at $700 \mathrm{pc}$, in agreement with the extremum of the mean square velocity components (see Fig. 6, left). The unexpected small value of $\alpha_{y y}$ from our approximations could be explained by the fact that the nonlinear flows of gas responsible for the local dynamos are not always directly connected to the large-scale magnetic field amplification (see Brummell et al. 2001, Sect. 1) or that the applied classical approximation of the dynamo coefficient $\alpha$ (Eq. (5), Moffatt 1978) is only the kinetic part of the full quantity taking into account also its magnetic part (see Kleeorin et al. 2003; Blackman \& Brandenburg 2002; Rogachevskii \& Kleeorin 2001; Kleeorin $\&$ Rogachevskii 1999). This problem will be a subject of our further considerations. To better understand the physics of the dynamo coefficients we plan also to perform future calculations with the initial magnetic field inclined to the azimuthal direction in order to get the radial component of the field, which is more realistic for galactic disks. The shearing process connected to the presence of the differential rotation will be included as well. Brandenburg \& Donner (1997) found that 
shearing was the most important process to study, as it had a profound effect on the sign and value of $\alpha_{\phi}$.

In the classical dynamo theory $\alpha_{z z}$ should be negative in the upper part of the galactic disk. Our model shows that the sign can change in time and this was also obtained by Brandenburg \& Donner (1997) in the calculations of the localized ISM with the assumptions of quenching and shearing. Brandenburg et al. (1995) from the cube simulations also get negative values of $\alpha_{\phi}$ in the upper disk plane. They conclude that the effect of a single eddy cannot be explained in a static description of the so-called "Parker loop", but this process should take into account dynamical effects of the loops growing in the $Z$ direction, which is a case in our model. In our opinion the EMF force, which is changing over time, could give such results in a turbulent medium even with quite strong helicity. Theoretically it is known that the components of $\hat{\alpha}$ could have an opposite sign to that expected, if the line of force is tangled more than $\pi$ during one turbulence life time (Parker 1979). Two complicated terms in every $\hat{\alpha}$ coefficient (Eq. (5)) implies that the real behavior of the dynamo coefficients is more complex than one could expect from classical dynamo models (Ruzmaikin et al. 1988), so a static explanation is no longer valid. In a later paper Brandenburg \& Schmitt (1998) showed that due to the magnetic buoyancy the $\alpha$-effect appeared to be positive in the northern hemisphere, however they computed its value only during the growing phase of instability.

For the first time in studies concerning the galactic dynamo we obtain two dynamo coefficients connected with the buoyancy mechanism: $\alpha_{y x}$ and $\alpha_{x y}$. Both experiments: B1 and D1 yield quite different time evolutions of these quantities (see Fig. 5). This fact is in agreement with our expectations that the small-scale gas motions are highly anisotropic and the antisymmetric part of $\alpha_{y x}^{A}$ exists (given by Eq. (6)). The models presented so far (e.g. Ziegler 1996; Brandenburg \& Donner 1997; Brandenburg \& Schmitt 1998) always assumed that turbulences were isotropic and $\alpha_{x y}=\alpha_{y x}$. The component $\alpha_{y x}^{A}$ is responsible for magnetic field transport outwards of the galactic disk in the $Z$ direction (see Ossendrijver et al. 2002; Ziegler 1996). So in our calculations instead of $V_{\text {esc }}=\alpha_{y x}$ we identify $V_{\text {esc }}$ with $\alpha_{y x}^{A}\left(V_{\mathrm{esc}}\right.$ as in the Ferrière papers). We obtain a quite small antisymmetric part of $\alpha_{y x}^{A}$ (the mean value is about $1 \mathrm{~km} \mathrm{~s}^{-1}$ ), by one order smaller than in the Ferrière (1993a,b, 1998) models. This fact opens the possibility of an additional magnetic field amplification in the disk, however we need stronger $\alpha_{y y}$ (see Sect. 1).

Our simulations result in a time-averaged value of the diffusion dynamo coefficient $\eta_{\mathrm{T}}$ of the order of $10^{26} \mathrm{~cm}^{2} \mathrm{~s}^{-1}$, which is in a very good agreement with the value obtained in the turbulent dynamo theory (Ruzmaikin et al. 1988).

The maximum of all dynamo coefficients and the square velocities is placed around $700 \mathrm{pc}$, where all the quantities have the highest peaks. This fact is connected to the character of the Parker instability process which has high extension above the disk plane, as was also found by Moss et al. (1999).

The saturation time for the small-scale motion flows in the model $\mathrm{B} 1$ with a low number of harmonic components of the velocity perturbation is by $100 \mathrm{Myr}$ shorter than for the run D1.
This fact remains in agreement with our expectations that a smaller number of perturbations should be more rapidly dissipated. A smaller number of small-scale gas motions in the beginning also gives the possibility of a slightly higher growth of the dynamo tensor components for the case B1 than D1 (see Tables 1 and 2, respectively).

The results of the computations of the model B2, with a two times weaker initial magnetic field than the models B1 and D1, shows that all selected dynamo components react differently to the magnetic field strength change (see also Ziegler 1996; Blackman \& Brandenburg 2002; Ossendrijver et al. 2001, 2002). The coefficients $\alpha_{x x}, \alpha_{x y}$ and $\alpha_{y x}^{A}$ reach somewhat higher maxima if we decrease the initial magnetic field. The rest of the dynamo coefficients decrease their value if the weaker initial magnetic field is adopted. These results are partly in agreement with results obtained by Ziegler (1996), in which the antisymmetric part of $\hat{\alpha}$ is higher than the rest of coefficients for the experiment with decreasing strength of the initial magnetic field.

\section{Conclusions}

In the present paper we have demonstrated that the Parker instability developing in the local galactic medium leads to flows giving rise to a substantial $\alpha$-effect. Our model allows for the time and space evolution (along the $Z$ direction) of the chosen dynamo coefficients defined as the tensors. This is a new and important way to obtain realistic dynamo coefficients and their role in the mean magnetic field evolution in galaxies and other astrophysical bodies (see also Blackman \& Brandenburg 2002). Our key results can be summarized as follows:

1. The numerically computed EMFs obtained from smallscale gas motion averaged in planes parallel to the galactic one evolve in time with strong fluctuations of all coefficients of the dynamo tensor.

2. The time-averaged values of $\alpha_{x x}$, as well as the peak value of $\alpha_{z z}$, are higher than in the SN numerical models, especially if quenching is taken into account. $\alpha_{z z}$ oscillates between positive and negative values. The time-averaged mean value of $\alpha_{y y}$, which is crucial in the large-scale dynamo process, has a much smaller mean value than the first described coefficients. The future inclusion of the radial component of the magnetic field and the differential rotation in our model could probably increase this component of the $\hat{\alpha}$-tensor. For the first time in galactic models two dynamo coefficients connected with the buoyancy force are calculated: $\alpha_{x y}$ and $\alpha_{y x}$. Both quantities show quite different time evolution, which means that the small-scale gas motions are fully anisotropic and it is necessary to compute $\alpha_{y x}^{A}$ (not only $\alpha_{y x}$ as in other simulations). The small value of $\alpha_{y x}^{A}$ obtained causes that the buoyancy mechanism slowly takes the magnetic field away from the galactic plane.

3. In both experiments the time-averaged mean value of the diffusion coefficient is of the order of $10^{26} \mathrm{~cm}^{2} \mathrm{~s}^{-1}$, in good agreement with the classical dynamo theory. 
4. The model with a smaller number of harmonic components of the velocity perturbations exhibits a shorter saturation time (by $100 \mathrm{Myr}$ ) than the experiment with more components.

5. The value of the dynamo coefficients have their peaks at about 700 pc above the disk plane, resulting in a large vertical extent of the dynamo coefficients.

6. The dynamo coefficients react in a different way to the change of the initial magnetic field strength, as was predicted in earlier papers (Ziegler 1996; Blackman \& Brandenburg 2002)

Acknowledgements. The author expresses her gratitude to Dr. Michał Hanasz, Dr. Detlef Elstner, Dr. Katia Ferrière, Dr. Marian Soida and Prof. Marek Urbanik for helpful discussions, constructive comments and suggestions. This work was partly supported by the Polish Committee for Scientific Research (KBN) (from the grants PB 0404/P03/2001/20 and PB 0249/P03/2001/21).

\section{References}

Blackman, E. G., \& Brandenburg, A. 2002, ApJ, 579, 359

Brandenburg, A., Nordlund, A., Stein, R. F., \& Torkelsson U. 1995, ApJ, 446, 741

Brandenburg, A., \& Donner, K. J. 1997, MNRAS, 288, L29

Brandenburg, A., \& Schmitt, D. 1998, A\&A, 338, L55

Brummell, N. H., Cattaneo, F., \& Tobias S. M. 2001, Proc. NATO Advanced Research Workshop Dynamo and Dynamics, a Mathematical Challenge (Kluwer Academic Publishers), 145

Brummell, N. H., Cattaneo, F., \& Tobias, S. M. 1998, Phys. Lett. A, 249,437

Choudhuri, A. R. 1992, A\&A, 253, 277

Chyży, K. T., Beck, R., Kohle, S., Klein, U., \& Urbanik, M. 2000, A\&A, 356, 757

Chyży, K. T., Knapik, J., Bomans, D. J., et al. 2003, A\&A, submitted

Covas, E., Tavakol, R., Tworkowski, A., \& Brandenburg, A. 1998, A\&A, 329, 350

Covas, E., Tworkowski, A., Brandenburg, A., \& Tavakol, R. 1997, A\&A, 317, 610

Ferrière, K. M 1993a, ApJ, 404, 162

Ferrière, K. M 1993b, ApJ, 409, 248

Ferrière, K. M 1995, ApJ, 441, 281

Ferrière, K. M 1996, A\&A, 310, 438

Ferrière, K. M. 1998, ApJ, 497, 759

Frisch, U., Pouquet, A., Leorat, J., \& Mazure, A. 1975, J. Fluid Mech., 68,769

Hanasz, M., \& Lesch, H. 1993, A\&A, 278, 561

Hanasz, M., \& Lesch, H. 1997, A\&A, 321, 1007

Hanasz, M., \& Lesch, H. 1998, A\&A, 332, 77

Hanasz, M., \& Lesch, H. 2000, ApJ, 543, 235

Hanasz, M., Otmianowska-Mazur, K., \& Lesch, H. 2002, A\&A, 386, 347 (Paper I)

Hoyng, P., Schmitt, D., \& Teuben, L. J. W. 1994, A\&A, 289, 265
Kleeorin, N., Moss, D., Rogachevskii, I., \& Sokoloff, D. 2003, A\&A, 400,9

Kleeorin, N., Moss, D., Rogachevskii, I., \& Sokoloff, D. 2002, A\&A, 387,453

Kleeorin, N., Moss, D., Rogachevskii, I., \& Sokoloff, D. 2000, A\&A, 361, L5

Kleeorin, N., \& Rogachevskii, I. 1999, Phys. Rev. E, 59, 6724

Konz, Ch., Wiechen, H., \& Lesch, H. 2000, Phys. Plasmas, 7, 5159

Korpi, M. J. 1999, Ph.D. Thesis, Oulun Yliopisto, Finland

Korpi, M. J., Brandenburg, A., Shukurov, A., Tuominen, I., \& Nordlund, A. 1999, ApJ, 514, L99

Kowal, G., Hanasz, M., \& Otmianowska-Mazur, K. 2003a, A\&A, 404, 533

Kowal, G., Hanasz, M., \& Otmianowska-Mazur, K. 2003b, Proc. of the JENAM 2002 (Kluwer Academic Publishers), in press

Krause, F., \& Rädler, K.-H. 1980, Mean-field Hydrodynamics and Dynamo Theory (Berlin: Akademieverlag)

Moffatt, K.-H. 1978, Magnetic field generation in electrically conducting fluids (Cambridge University Press)

Moss, D., Brandenburg, A., Tavakol R., \& Tuominen, I. 1992, A\&A, 265,843

Moss, D., Shukurov, A., \& Sokoloff, D. 1999, A\&A, 343, 120

Ossendrijver, M., Stix, M., Brandenburg, A., \& Rüdiger, G. 2002, A\&A, 394, 735

Ossendrijver, M., Stix, M., \& Brandenburg, A. 2001, A\&A, 376, 713

Otmianowska-Mazur, K., Hanasz, M., \& Kowal, G. 2003, Proc. of the JENAM 2002 (Kluwer Academic Publishers), in press

Otmianowska-Mazur, K., Chyży, K., Soida, M., \& von Linden, S. 2000, A\&A, 359, 29

Otmianowska-Mazur, K., Rüdiger, G., Elstner, D., \& Arlt, R. 1997, GAFD, 86, 229

Parker, E. N. 1992, ApJ, 401, 137

Parker, E. N. 1979, Cosmical Magnetic Fields (Clarendon Press Oxford)

Parker, E. N. 1966, ApJ, 145, 811

Pouquet, A., Frisch, U., \& Lèorat, J. 1976, J. Fluid Mech., 77, 321

Rädler, K.-H. 1980, AN, 301, 101

Rogachevskii, I., \& Kleeorin, N. 2001, Phys. Rev. E, 64, 056307

Ruzmaikin, A. A., Shukurov, A. M., \& Sokoloff, D. D. 1988, Magnetic Fields of Galaxies, Astrophys. and Space Science Lib. (Dordrecht: Kluwer Academic Publishers)

Schmalz, S., \& Stix, M. 1991, A\&A, 245, 654

Schmitt, D. 1993, Proc. of IAU Symp. The Cosmic Dynamo, ed. F. Krause, K.-H. Rädler, \& G. Rüdiger (Kluwer Academic Publishers)

Stone, J. M., \& Norman, M. L. 1992a, ApJS, 80, 753

Stone, J. M., \& Norman, M. L. 1992b, ApJS, 80, 791

Tanuma, S., Yokoyama, T., Kudoh, T., et al. 1999, PASJ, 51, 161

Tanuma, S., Yokoyama, T., Kudoh, T., \& Shibata, K. 2001, ApJ, 551, 312

Ugai, M. 1992, Phys. Fluids B, 4, 2953

Widrow, L. M. 2002, Rev. Mod. Phys., 74, 775

Ziegler, U., Yorke, H. W., \& Kaisig, M. 1996, A\&A, 305, 114

Ziegler U. 1996, A\&A, 313, 448 